\title{
Inflammatory pain assessment in the arthritis of the temporomandibular joint in rats: A comparison between two phlogistic agents
}

\author{
Joana Cláudia Bezerra de Araújo ${ }^{\mathrm{a}}$, Delane Viana Gondim ${ }^{\mathrm{b}}$, André Luiz Cunha Cavalcante ${ }^{\mathrm{c}}$, \\ Mario Roberto Pontes Lisboa ${ }^{\mathrm{b}}$, Gerly Anne de Castro Brito ${ }^{\mathrm{b}}$, Mariana Lima Vale ${ }^{\mathrm{a}, \mathrm{b}, *}$ \\ a Pharmacology Post Graduation Program, Department of Physiology and Pharmacology, Federal University of Ceará, Brazil \\ b Morphofunctional Sciences Post Graduation Program, Department of Morphology, Federal University of Ceará, Brazil \\ ${ }^{c}$ Medical Sciences Post Graduation Program, Department of Clinical Medicine, Federal University of Ceará, Brazil
}

\begin{abstract}
A B S T R A C T
Temporomandibular joint (TMJ) disorders are a group of conditions that result in TMJ pain, which frequently limits basic daily activities. Experimental models that allow the study of the mechanisms underlying these inflammatory and pain conditions are of great clinical relevance. The aim of this study was to evaluate nociception, inflammation and participation of the macrophage/microglia cells in the arthritis of the TMJ induced by two phlogistic agents. 84 rats were divided into 2 groups: $\mathrm{Zy}$, which received zymosan intra-articularly, or $\mathrm{Cg}$, which received carrageenan intra-articularly. Mechanical nociception, total leukocyte influx to the synovial fluid and histopathological analyses were evaluated in the TMJ. The participation of macrophage/microglia located in trigeminal ganglia (TG) and in the subnucleus caudalis (V-SnC) was assessed immunohistochemically. Both agents induced mechanical hyperalgesia $6 \mathrm{~h}$ after the induction, but a more persistent algesic state was perceived in the $\mathrm{Cg}$ group, which lasted for $120 \mathrm{~h}$. Even though both groups presented increased leukocyte influx, the Zygroup presented a more intense influx. Zymosan recruited resident macrophage in the trigeminal ganglia $24 \mathrm{~h}$ after the injection. In the V-SnC, the group $\mathrm{Cg}$ presented a more prolonged immunolabeling pattern in comparison with the group Zy. It can be concluded that zymosan induced a more intense infiltrate and peripheral nervous changes, while $\mathrm{Cg}$ lead to a moderate TMJ inflammation with prominent changes in the $\mathrm{V}$-SnC.
\end{abstract}

\section{Introduction}

Disorders regarding orofacial pain are debilitating conditions that affect the head, face and/or neck region of around $90 \%$ of the population and might have physical or psychological origins (RomeroReyes \& Uyanik, 2014). With the exception of tooth-related pain, temporomandibular joint (TMJ) disorders are the main reason that lead people to seek treatment for orofacial pain, even though only $10-20 \%$ of those patients are properly treated (Nassif, Al-Salleeh, \& Al-Admawi, 2003; Von Korff, Dworkin, Le Resche, \& Kruger, 1988).

Regardless of the nature of the orofacial pain, a general mechanism for the conduction of a nociceptive impulse might be explained. A local noxious stimulus depolarizes the peripheral free nerve endings of a primary neuron and the action potential conducted to the subnucleus caudalis (V-SnC), where the first synapse takes place. Consequently, the impulse is finally conducted to the ventral posteromedial nucleus of the thalamus and to the somatosensory cortex (Takemura, Sugiyo, Moritani, Kobayashi, \& Yonehara, 2006).
For an extensive period of time, the physiopathology of pain relied mainly on the synaptic role of the neuron (Hucho \& Levine, 2007). However, it is now clear that macrophage/microglia cells exert an important function within the pain process, modulating neuronal synaptic function and neuronal excitability by various mechanisms (Halassa, Fellin, Takano, Dong, \& Haydon, 2007). Astrocytes, microglia and satellite glial cells were shown to be capable of changing the production of cytokines and chemokines in degenerative inflammatory conditions (Souza et al., 2013; Suter, Wen, Decosterd, \& Ji, 2007) and microglial cells, different from other mononuclear macrophages associated to the central nervous system (CNS), interact with neurons, synapses and other glial cells in pathological conditions in the CNS parenchyma (Mosser, Baptista, Arnoux, \& Audinat, 2017). Microglial activation increases proliferation, phagocytic activity and the release of pro-inflammatory mediators, which activate the neurons in an individualized manner, favoring nociception (Milligan \& Watkins, 2009). It was shown that TMJ inflammation induced by Complete Freund's Adjuvant (CFA) activates glial and immune cells in both the TG and in

\footnotetext{
* Corresponding author at: Department of Physiology and Pharmacology, Faculty of Medicine, Federal University of Ceará - UFC, Rua Cel. Nunes de Melo, 1127, Rodolfo Teófilo, 60430-270 Fortaleza, CE, Brazil.

E-mail address: mariana.vale@pq.cnpq.br (M.L. Vale).
} 
the V-SnC (Villa et al., 2010). Evidence suggests that glial cells of sensory ganglia also participate in the development and maintenance of chronic pain condition (McMahon \& Malcangio, 2009).

Even though several experimental models are used for studying disorders of the TMJ, little is known about the nociceptive mechanism and the changes in the central and peripheral nervous systems among the different models. A proper study of the pathogenesis of induced arthritis models is then indicated in order to elucidate the choice of the most accurate experimental model depending on the aims of the study. Therefore, the purpose of this study was to evaluate the nociception, inflammation and the participation of macrophage/microglial cells in two different models of TMJ arthritis.

\section{Materials and methods}

\subsection{Sample size}

For calculating the sample size, Wistar rats (180-200 g) were considered as the study unit and the head withdrawal threshold was considered the primary outcome. The sample size was determined in order to recognize a significant difference of $20 \%$ from the baseline in the primary outcome with a power of $80 \%$. The standard deviation was established at $15 \%$ with a $95 \%$ interval of confidence. Therefore, a sample size of 7 animals was required for each experimental group.

\subsection{Animals}

The experimental protocols for this study was approved and analyzed by the Ethics Committee on Animal Research at Federal University of Ceará - UFC (protocol 27/10) and considered in compliance with the ethical principles of animal experimentation, as well as standards for the didactic-scientific practice of vivisection and the National Institutes of Health guide for the care and use of laboratory animals

Eighty-four adult male rats (Rattus norvegicus, albinus, Wistar), weighing between $180 \mathrm{~g}$ and $200 \mathrm{~g}$, were provided by the Animals Facility in the Department of Physiology and Pharmacology of the Federal University of Ceará. The animals were housed in proper plastic cages, with no $>6$ animals per cage, with solid food and water ad libitum in a room with a $12 \mathrm{~h}$ light/dark cycle at a temperature of $22 \pm 2{ }^{\circ} \mathrm{C}$.

The animals were then divided into 2 major groups: group $\mathrm{Zy}$, which received a $40 \mu \mathrm{l}$ intra-articular injection of a $5 \%$ zymosan solution, and group $\mathrm{Cg}$, which received a $10 \mu \mathrm{l}$ intra-articular injection of a $5 \%$ carrageenan solution, following well established models for TMJ arthritis (Cavalcante et al., 2013; Chaves et al., 2011).

\subsection{Induction of the TMJ arthritis}

The animals were anesthetized by an intraperitoneal injection of a solution of Ketamine $(70 \mathrm{mg} / \mathrm{kg}$ ) and Xylazine $(10 \mathrm{mg} / \mathrm{kg})$ and received an intra-articular (i.art.) injection of zymosan $(2 \mathrm{mg}, 40 \mu \mathrm{l}$ total volume) or $5 \% \mathrm{Cg}$ solution $(500 \mu \mathrm{g}$ per articulation; $10 \mu \mathrm{l})$ dissolved in sterile saline into the supra-discal space of the left TMJ (ipsilateral) using a microsyringe (Hamilton model 705RN; Hamilton, Reno, NV, USA) coupled to a 30-gauge needle (BD, Franklin Lakes, NJ, USA). As a control procedure, another group of animals was intraarticularly injected with saline unilaterally in the left joint.

In order to properly locate the TMJ capsule, the animals' mandibles were manipulated and the needle was inferiorly inserted to the posterior inferior border of the zygomatic arch. When a resistance was found, the mandible was once again manipulated to confirm the capsule location. The accuracy of the injection was confirmed by the lack of resistance when the needle passed through the capsule (Chaves et al., 2011; Gondim et al., 2012).

\subsection{Evaluation of the mechanical sensitivity}

The nociceptive threshold of the animals was assessed by the intensity of pressure applied to the left TMJ area that generated a reflex response (head withdrawal threshold; HWT), using an electronic Von Frey equipment (Analgesímetro Digital, Insight, Ribeirão Preto, SP, Brazil). The facial areas to be tested around the TMJ were carefully shaved, and the animals were placed into individual plastic cages $45 \mathrm{~min}$ before beginning the tests. During the 5 days preceding the experimental period, the animals were adapted for manipulation in order to properly assess their nociceptive threshold without stressing the animals. The transducer was perpendicularly applied to the central area of the TMJ region with a gradual increase in pressure. The stimulus was automatically discontinued, and the intensity was recorded when the head was withdrawn. The end-point was characterized by the removal of the head in a clear flinch response after head withdrawal (Denadai-Souza et al., 2010).

In both groups, the HWT was measured before the induction of the arthritis of the TMJ and 6, 24, 48, 72 and $120 \mathrm{~h}$ after the administration of the phlogistic agents.

\subsection{Total leukocyte count in the synovial fluid}

Our group has previously shown that the 6th hour is marked by an intense inflammatory infiltrate followed by the injection of both $\mathrm{Zy}$ and Cg (Cavalcante et al., 2013; Chaves et al., 2011). Therefore, $6 \mathrm{~h}$ after the induction of TMJ arthritis, a subgroup of the animals was euthanized by intracardiac perfusion with $40 \mathrm{ml}$ of saline solution followed by $40 \mathrm{ml}$ of $4 \%$ paraformaldehyde (PFA). The skin and the temporal muscle were deflected and the articular wash was performed by injecting and aspirating $100 \mu \mathrm{l}(2 \times 50 \mu \mathrm{l})$ of saline solution with heparin $(5 \mathrm{U} / \mathrm{ml})$ using ultrafine insulin syringes. A sample of $20 \mu \mathrm{l}$ from the articular wash was mixed with $380 \mu l$ of Turk's solution and the cells were counted under light microscopy with a Newbauer's Chamber.

The synovial fluid of the TMJ of saline group was collected, following the same procedures, in order to compare the leukocyte count within these animals with the animals treated with the phlogistic agents.

\subsection{Histopathological analysis}

A histopathological analysis of the TMJs was performed in the peak of pain for each model. The rats were euthanized, the facial skin was excised, and the temporal muscle that overlays the TMJ was dissected. After the TMJ and periarticular tissue were excised, the tissues were fixed in $10 \%$ neutral buffered formalin for $24 \mathrm{~h}$, demineralized in $10 \%$ ethylenediaminetetraacetic acid, embedded in paraffin and sectioned along the long axis of the TMJ. Sections $(4 \mu \mathrm{m})$ showing the mandibular condyle, articular cartilage, articular disc, synovial membrane, periarticular tissue, and skeletal muscle periarticular tissue were evaluated under light microscopy by a certified histotechnologist (G.A.B.), who evaluated the inflammatory infiltrate influx, as well as the integrity of the articular tissues (synovial membrane, mandible condyle and articular cartilage).

\subsection{Immunofluorescence analysis of ionized calcium binding adaptor molecule-1 (Iba-1) expression in the trigeminal ganglion}

After the animals were euthanized, their brains were removed and the left trigeminal ganglia were easily seen in the intern aspect of the neurocranium. The ipsilateral TG was removed and fixed for $2 \mathrm{~h}$ in $4 \%$ PFA. It was then cryoprotected in $30 \%$ sucrose solution for $72 \mathrm{~h}$, embedded in Tissue-Tek ${ }^{\circledast}$ and froze-stored at $-80{ }^{\circ} \mathrm{C}$ until further evaluation. Sixteen $\mu \mathrm{m}$-thick histological sections were mounted in poly-Llysine microscope slides and went through antigenic retrieval in citrate 
buffer ( $\mathrm{pH}$ 6.0) for $15 \mathrm{~min}$ at $95{ }^{\circ} \mathrm{C}$. Then, the sections were incubated overnight $\left(4{ }^{\circ} \mathrm{C}\right.$ ) with primary rabbit anti-Iba-1 (Wako Chemicals, Richmond, VA, USA) diluted 1:400 in phosphate buffered saline (PBS) and $5 \%$ bovine serum albumin (BSA). The slides were then washed with PBS and incubated for $90 \mathrm{~min}$ in secondary goat anti-rabbit IgG combined with Alexa fluor 594 (Invitrogen, CA, USA) diluted 1:400 in 5\% BSA. Finally, the sections were washed with PBS, incubated for $10 \mathrm{~min}$ with 4',6-diamidino-2-phenylindole (DAPI) and mounted with a specific medium for fluorescent staining (Fluoromount, Sigma-Aldrich, MO, USA).

The sections were visualized by confocal microscopy (Olympus f1000) by a certified operator (M.L.V.) and the area marked in red was quantified using the appropriate image processing software (Fiji, NIH, WI, USA).

\subsection{Immunohistochemical analysis of Iba-1-positive cells in the V-SnC}

After the euthanasia of the animals, the brain and the brainstem were dissected and positioned in a specially confectioned acrylic matrix (Insight, Ribeirão Preto, São Paulo, Brazil). The trigeminal nuclei area was dissected and fixated/set for $2 \mathrm{~h}$ in $4 \%$ PFA. It was then cryoprotected in $30 \%$ sucrose solution for $72 \mathrm{~h}$, embedded in Tissue-Tek ${ }^{\circledast}$ and froze-stored at $-80{ }^{\circ} \mathrm{C}$ until further evaluation. The sections used in this study were cut $-10.5 \mathrm{~mm}$ to $-14.04 \mathrm{~mm}$ from bregma (Gondim et al., 2012) The samples were then histologically processed and embedded in paraffin as mentioned before. Sections of $4 \mu \mathrm{m}$ sections were mounted in poly-L-lysine microscope slides. The antigenic retrieval was performed similarly to the trigeminal ganglia slides. Endogen peroxidase was blocked with $3 \%$ hydrogen peroxide for $20 \mathrm{~min}$. The sections were washed with $\mathrm{PBS}$ and incubated overnight $\left(4^{\circ} \mathrm{C}\right)$ with a primary rabbit anti-Iba-1 antibody (Wako Chemicals, Richmond, VA, USA). Then, the sections were washed with PBS and incubated for $30 \mathrm{~min}$ with a biotinylated goat anti-rabbit IgG antibody, rewashed with PBS and incubated for $30 \mathrm{~min}$ with avidin-biotin-horseradish peroxidase conjugate (Strep ABC Complex; Santa Cruz Biotechnology, CA, USA) according to the manufacturer's protocol. The immunolabeling was visualized with a cromogen 3, 3'-diaminobenzidine (DAB, Santa Cruz Biotechnology, CA, USA) and counterstained with Mayer's hematoxylin. Negative control for the reactions was performed following the same protocol, except for the incubation with the primary antibody. The sections were dehydrated and cleared in series of alcohol and xylol and cover-slipped. The Iba-1 immunolabeling pattern was qualitatively analyzed by an experienced histolotechnologist (M.L.V.).

\subsection{Statistical analyses}

All data were grouped and expressed as means \pm standard error of the mean. Normality was evaluated by Shapiro-Wilk's test. Outliers were removed. The Student's $t$-test or one-way ANOVA were used to analyze two or three groups, respectively. The two-way ANOVA with Tukey pos hoc test was used to analyze the interaction between Zy and $\mathrm{Cg}$ groups at the same time. Statistical analyses were performed using the GraphPad Prism 6 (GraphPad Prism software, La Jolla, CA, USA) computer software programs. Probability level ( $p$ value $)<0.05$ was assumed.

\section{Results}

\subsection{Effects of $\mathrm{Cy}$ and $\mathrm{Cg}$ in mechanical sensitivity}

In both groups, the injection of the phlogistic agents induced a reduction in the HWT in the 6th hour. The hyperalgesic response persisted for $24 \mathrm{~h}$ among the animals that received $\mathrm{Zy}$ injection, while the effect lasted for $120 \mathrm{~h}$ among the animals that received intra-articular $\mathrm{Cg}$. Intergroup evaluation showed that $\mathrm{Cg}$ induced a significantly more intense hyperalgesia in all periods after the injection $(p<0.001)$,



Fig. 1. Temporal evaluation of the mechanical nociception (head withdraw threshold HWT) with comparison among the groups. The phlogistic agents were injected after the baseline evaluation $(0 \mathrm{~h})$. Data are represented as mean \pm standard error of the mean. ${ }^{*} p<0.001$, (Two way ANOVA).

except in the 6th hour, when it was statistically similar to the Zy group $(p>0.05$; Fig. 1).

\subsection{Leukocyte influx after $\mathrm{Zy}$ or Cg injection in the TMJ}

Seeing that the peak of the hyperalgesic response was in the 6th hour for both phlogistic agents, a cell count was performed at this period. A severe influx was observed in group Zy $\left(12.25 \times 10^{6}\right.$ cells/ $\mathrm{ml}$ ) and it was statistically different from the one observed in the naïve group $\left(0.55 \times 10^{6}\right.$ cells $\left./ \mathrm{ml} ; p<0.01\right)$ (Fig. $\left.2 \mathrm{~A}\right)$. In the $\mathrm{Cg}$ group, a moderately increased leukocyte count was found $\left(2.15 \times 10^{6}\right.$ cells $\left./ \mathrm{ml}\right)$ in comparison with the same saline group ( $p<0.01$; Fig. 2B). The data of the infiltrate influx were further normalized to the saline values, in order to evaluate the increase of the infiltrate influx. The $\mathrm{Cg}$ group showed a 3.91-fold increase in comparison with the saline animals, while the Zy group showed a 22.28-fold increase.

\subsection{Histopathological evaluation of the TMJ}

The TMJ of the animals was removed in the peak of pain after the induction the arthritis in both groups. By way of comparison, saline group had their TMJ histologically processed (Fig. 3A-B). A neutrophilic infiltrate and edema in the synovial membrane could be observed in the TMJ and the periarticular tissues of both Zy (Fig. 3C-D) and $\mathrm{Cg}$ groups (Fig. 3E-F). These characteristics were more evident within the Zy group, in which a much more intense inflammatory infiltrate was observed. In this group, the edema of the synovial membrane led to tissue disorganization and increased thickness of the synovial membrane. Moreover, erythrocytes were evident in the TMJ of the animals treated with $\mathrm{Cg}$, indicating a possible micro haemorrhage.

\subsection{Iba-1 expression in the trigeminal ganglion}

The immunofluorescence assay showed that $\mathrm{Zy}$ and $\mathrm{Cg}$ administration induced an increased immunoreactivity for Iba- 1 in the TG of the animals (Fig. 4). However, a significant increase in immunoreactivity was only observed in the Zy group 24 after Zy injection in comparison to the group receiving saline injection ( $p<0.05$; Fig. 5). No statistical difference was observed between the $\mathrm{Zy}$ and $\mathrm{Cg}$ groups over the time $(p>0,05)$.

\subsection{Iba-1 expression in the $\mathrm{V}-\mathrm{SnC}$}

A sole dose of $\mathrm{Zy}$ or $\mathrm{Cg}$ was capable of increasing the immunolabeling pattern of Iba- 1 in the V-SnC of the animals, in comparison with saline group (Figs. 6 and 7). A more intense immunoreactivity in the Zy group was observed 24 and $48 \mathrm{~h}$ after the administration of the Zy (Fig. 6G-I). An increased immunolabeling 

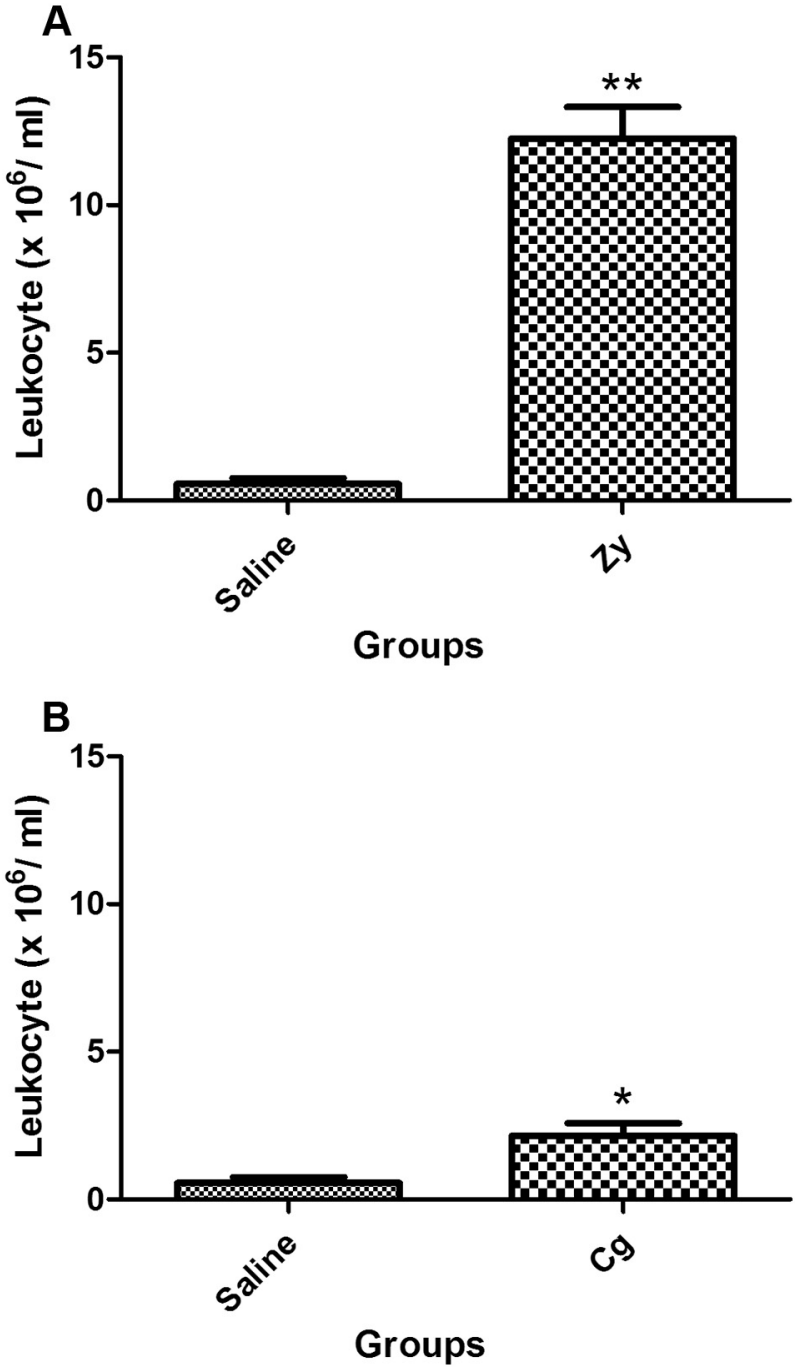

Fig. 2. Total leukocyte influx to the synovial fluid of the animals the groups $\mathrm{Zy}$ (Fig. 2A) and $\mathrm{Cg}$ (Fig. 2B). For comparison, the synovial wash of animals treated with intra-articular saline. Data are represented as mean \pm standard error of the mean. ${ }^{*} p<0.05$, when compared to the saline group; ${ }^{\#} p<0.05$, when compared to the Cg group (Student's $t$-test).

within the Cg group was observed up to the 72nd hour (Fig. 7D-L). Moreover, it was interesting to find that both drugs led to morphological differentiations in the microglia, inducing them to a more swollen and branched state. The administration of $\mathrm{Cg}$ seemed to lead to a more intense immunolabeling pattern than the administration of $\mathrm{Zy}$ in the $\mathrm{V}$ SnC.

\section{Discussion}

TMJ disorders are a set of conditions that lead to orofacial pain and have a highly negative impact on life quality (Ahmed, Mustafa, Catrina, \& Alstergren, 2013; Ahola et al., 2015). The TMJ inflammation influences the central processing of nociceptive input in the trigeminal pathway. In the presence of inflammatory process, monocyte derived macrophages invade injured tissue and release a complex array of cytokines, chemokines and growth factors such as NGF, transforming the nociceptor phenotype to pathophysiologic states of persistent nociceptor activation, lowered firing thresholds and/or exaggerated response properties (Guan, Hellman, \& Schumacher, 2016). As these conditions might have many etiologic factors, different experimental models try to properly mimic their clinical outcomes. Therefore, the aim of this study was to evaluate the nociceptive and inflammatory parameters and macrophage/microglial cells activation on inflammatory orofacial pain due to TMJ arthritis induced by two different phlogistic agents.

Initially, both agents succeeded in inducing hyperalgesia in the TMJ, as observed by the mechanical nociception assay. Both Zy and Cg induced a decrease in the HWT by the 6th hour, as previously shown (Cavalcante et al., 2013; Chaves et al., 2011; da Conceição Rivanor et al., 2014; Denadai-Souza et al., 2010; do Val et al., 2014). Signs of articular inflammation were also found in both groups, as evidenced by the histopathological analysis and the leukocyte count in the synovial wash. Indeed, studies (Cunha, Poole, Lorenzetti, \& Ferreira, 1992; Gondim et al., 2012; Ribeiro et al., 2000) have shown that local administration of $\mathrm{Cg}$ and $\mathrm{Zy}$ lead to a release of proinflammatory cytokines, such as tumor necrosis factor- $\alpha$ (TNF- $\alpha$ ), interleukins(IL)- $1 \beta$ and 8 , and cyclooxygenase-2, resulting in inflammatory outcomes and sensitization of nociceptive neurons. Moreover, both agents induced peripheral and central changes in the TG and the V-SnC, respectively, which is in accordance with previous studies (Cavalcante et al., 2013; Gondim et al., 2012).

Regarding the mechanical nociception evaluation, the Zy group presented a significantly reduced HWT that lasted until the 24th hour after the administration of the drug. These data are in accordance with other researchers (Chaves et al., 2011; da Conceição Rivanor et al., 2014) who found that Zy-induced TMJ arthritis led to increased mechanical hyperalgesia in rats up to the 6th hour. The $\mathrm{Cg}$ group, on the other hand, presented a more persistent reduction in the HWT, which lasted until the end of the experimental period. In fact, it has been shown that $\mathrm{Cg}$ is able to influence the mechanical threshold in rats' TMJ up to 7 days after the intra-articular injection (Denadai-Souza et al., 2010).

Studies have shown that the enhanced hyperalgesia induced by $\mathrm{Cg}$ and $\mathrm{Zy}$ is dependent on inflammatory infiltration, increased cytokines release and activation of the nuclear factor $\kappa$ B (Chaves et al., 2011; da Conceição Rivanor et al., 2014; Guerrero et al., 2012; Pena-dos-Santos et al., 2009; Ruiz-Miyazawa et al., 2015). In fact, our data showed an intense inflammatory infiltrate in the synovial fluid of both groups after the injection of the phlogistic agents. It is interesting to see that a much higher leukocyte count can be observed within the Zy group, indicating a more intense influx in the first $6 \mathrm{~h}$ after the administration, in comparison with the animals that received $\mathrm{Cg}$.

In the qualitative histopathological analysis, both groups presented interstitial edema, evidenced by tissue disorganization and increased thickness of the synovial membrane, and a moderate to intense inflammatory infiltrate. It was noticed that these characteristics were more evident in the Zy group. Intra-articular injection of Zy leads to a higher vascular permeability in the TMJ, thus culminating in articular edema and intense cellular migration to the articular tissues and the synovial fluid, as found in this study (Gondim et al., 2012).

In the temporomandibular disorders, neuropeptides stimulate synovial tissues to produce several cytokines such as TNF- $\alpha$ and IL-1 $\beta$ and IL-6, and these are responsible for activating neurons and glia of synovial membrane at the bilaminar regions of the TMJ (Liu et al., 2014). Many recent studies have shown that macrophage/microglial cells play an important role in the response to pain by controlling the synaptic environment (Clark et al., 2007; Mika, 2008; Sweitzer, Schubert, \& DeLeo, 2001). In the present study, it was found that intraarticular injection of zymosan induced an increased immunolabeling of Iba-1 in the trigeminal ganglion at $24 \mathrm{~h}$. These data corroborate with Cady, Glenn, Smith, and Durham (2011), which showed that TMJ inflammation increases p-ERK staining intensity in satellite glial cells in trigeminal ganglia at $24 \mathrm{~h}$ (Cady et al., 2011). It has been shown that ERK stimulates the synthesis and secretion of cytokines from glial cells, which promote the state the hyperexcitability of neurons. CFA injection into the TMJ induced a strong up-regulation of ED1 (a specific marker for activated macrophages) in the ipsilateral trigeminal ganglion, but there was no increase in either the number or the average cell size of 





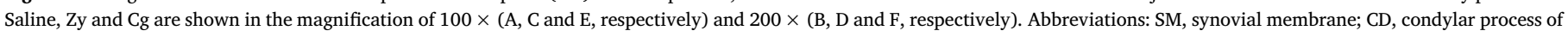
mandible.

Iba1 $^{+}$macrophages (Villa et al., 2010), thus this phlogistic agent did not cause macrophage infiltration into the trigeminal ganglion. However, to the best of our knowledge, this is the first evidence that $\mathrm{Zy}$ induced inflammatory orofacial pain leads to increased immunolabeling of Iba-1 in the trigeminal ganglion.

It was observed that a peripheral paw inflammation induced by both $\mathrm{Cg}$ and Zy caused a central sensitization, marked by microglial cells activation in the spinal cord (Vega-Avelaira, Ballesteros, \& LópezGarcía, 2013). Similarly, we found that peripheral TMJ inflammation with both agents led to an increased immunolabeling of Iba-1 in the V$\mathrm{SnC}$ when compared to the saline group. These data are corroborated by other studies (Cady, Denson, Sullivan, Durham, 2014; Villa et al., 2010), in which arthritis of the TMJ induced by CFA induced an increased immunolabeling of Iba- 1 in the V-SnC. However, this is the very first evidence that both $\mathrm{Zy}$ and $\mathrm{Cg}$ also induce the aforementioned effect.

It is important to emphasize that a remarkable difference was found in Iba-1 labeling among the groups. Zy induced a more prominent change in Iba-1 immunoreactivity in the TG. Resident macrophage in the trigeminal ganglion may secrete inflammatory mediators, such as TNF- $\alpha$, IL- $1 \beta$, IL- 6 and bradykinin, which modify the resting membrane potential of the primary afferent neuron, making it more positive, a process called peripheral sensitization (Villa et al., 2010; Franceschini et al., 2013). Such process might be responsible for the primary hyperalgesia observed in the Zy stimulated rats. Carrageenan, however, leads to a more intense alteration in Iba-1 positive cells in the V-SnC. Microglial cells exert an important role in the homeostasis of neurons in the central nervous system. These cells express many of the neurotransmitter receptors that are found in astrocytes and neurons (Pocock \& Kettenmann, 2007) and, once microglia become activated, they produce and release many substances that activate nearby astrocytes, microglia and neurons, which might lead to a central sensitization and chronic hyperalgesia observed in the Cg-stimulated group (Milligan \& Watkins, 2009). 


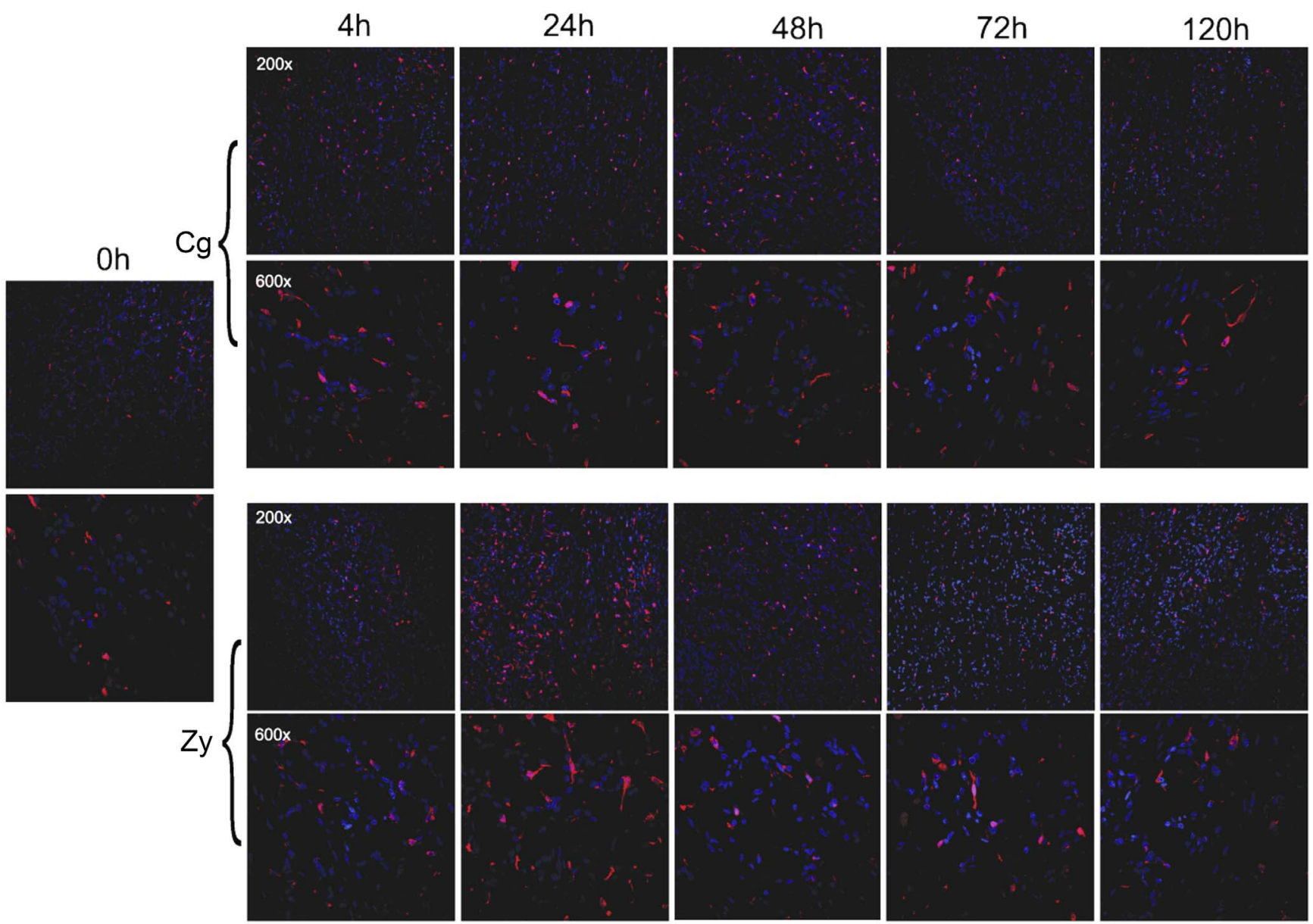

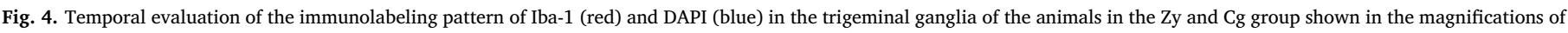
$200 \times$ and $600 \times$. (For interpretation of the references to colour in this figure legend, the reader is referred to the web version of this article.)

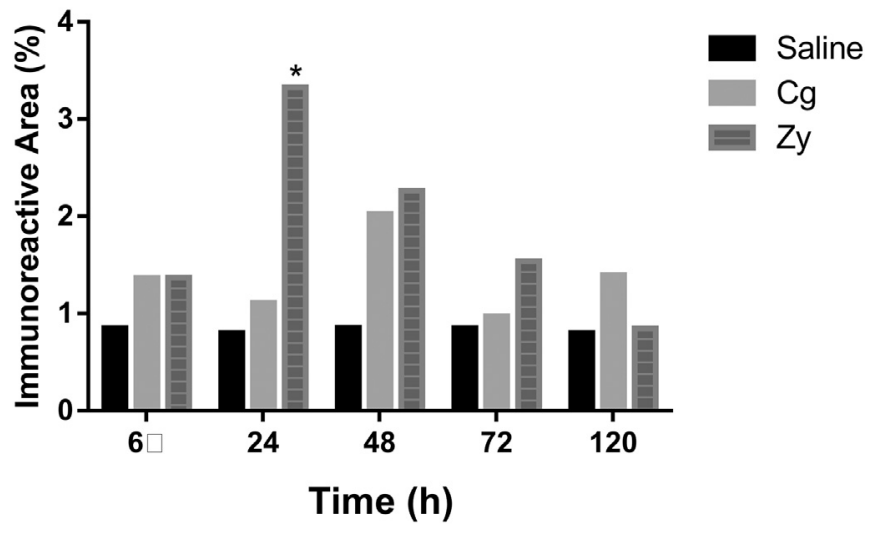

Fig. 5. Temporal quantification of the percentage of immunoreactive area of Iba-1 in the trigeminal ganglia of the animals in the Saline, $\mathrm{Zy}$ and $\mathrm{Cg}$ groups. Data are represented as mean \pm standard error of the mean. ${ }^{*} p<0.05$, when compared to the saline group (Two-Way ANOVA, Tukey).

In addition to the inherent limitations of experimental models, it is important to emphasize that disorders of the TMJ are multifactorial conditions influenced by the environment and the subject's response to psychological and physical stressor factors. This multifactorial nature hampers the development of experimental models that properly follow the clinical outcomes of the disorders. Nevertheless, the understanding of the physiopathology of TMJ arthritis is the key for the development of new approaches to treat orofacial pain induced by TMJ disorders.

\section{Conclusion}

In summary, even though both drugs induced orofacial pain, their peripheral and central nervous mechanisms were clearly different. To our understating, the preference for one or another phlogistic agent used in the model of TMJ arthritis must be based on the aim of the study. Zy-induced TMJ arthritis seemed to be more indicated to study the inflammatory aspects of the TMJ and the mechanisms of activation of the primary afferent, which means primary peripheral sensitization and cellular changes in the TG. On the other hand, the model of $\mathrm{Cg}$ induced TMJ arthritis is more representative for studies that aim to evaluate the persistent aspect of the orofacial hyperalgesia or the alterations in the central nervous system due to a peripheral TMJ inflammation.

\section{Conflicts of interest}

The authors declare no conflict of interest.

\section{Author contribution}

Joana C. de Araújo, André LuiCavalcante, Delane V Gondim e Mário $\mathrm{R}$ Lisboa have performed the experimental phase and the collection of samples.

Mario R. Lisboa has performed the statistical analysis of data.

Delane V. Gondim and Mario R. Lisboa have written the manuscript. Gerly A. Brito was responsable for the histologic evaluation and the interpretation of its data. 

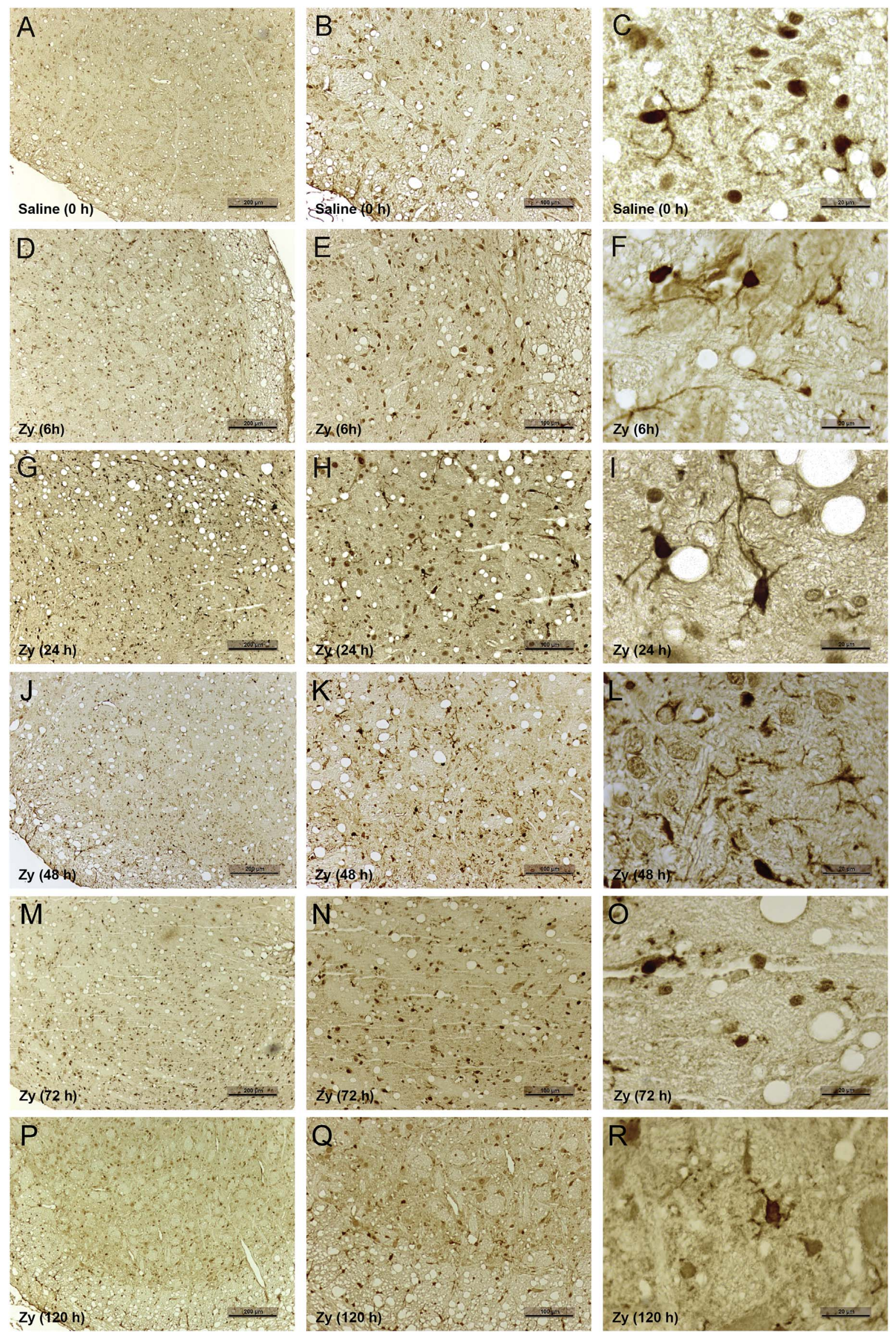

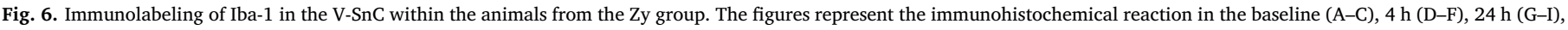
$48 \mathrm{~h}(\mathrm{~J}-\mathrm{L}), 72 \mathrm{~h}(\mathrm{M}-\mathrm{O})$ and $120 \mathrm{~h}(\mathrm{P}-\mathrm{R})$ after Zy injection in the magnifications of $40 \times(\mathrm{A}, \mathrm{D}, \mathrm{G}, \mathrm{J}, \mathrm{M}$ and $\mathrm{P}), 200 \times(\mathrm{B}, \mathrm{E}, \mathrm{H}, \mathrm{K}, \mathrm{N}$ and $\mathrm{Q})$ and $1000 \times(\mathrm{C}, \mathrm{F}, \mathrm{I}, \mathrm{L}, \mathrm{O}$ and R). 





$48 \mathrm{~h}(\mathrm{~J}-\mathrm{L}), 72 \mathrm{~h}(\mathrm{M}-\mathrm{O})$ and $120 \mathrm{~h}(\mathrm{P}-\mathrm{R})$ after $\mathrm{Cg}$ injection in the magnifications of $40 \times(\mathrm{A}, \mathrm{D}, \mathrm{G}, \mathrm{J}, \mathrm{M}$ and $\mathrm{P}), 200 \times(\mathrm{B}, \mathrm{E}, \mathrm{H}, \mathrm{K}, \mathrm{N}$ and $\mathrm{Q})$ and $1000 \times(\mathrm{C}, \mathrm{F}, \mathrm{I}, \mathrm{L}, \mathrm{O}$ and $\mathrm{R})$. 
Mariana L. Vale has designed this study and was coordinator of the research project.

\section{Acknowledgements}

The authors thank Mrs. Maria Silvandira França Pinheiro and Mr. Haroldo Pinheiro from the Department of Physiology and Pharmacology and Ms.

Maria do Socorro França Monte from the Department of Morphology, Faculty of Medicine, Federal University of Ceará, Brazil, for technical assistance.

\section{References}

Ahmed, N., Mustafa, H. M., Catrina, A. I., \& Alstergren, P. (2013). Impact of temporomandibular joint pain in rheumatoid arthritis. Mediators of Inflammation, 2013, 597419.

Ahola, K., Saarinen, A., Kuuliala, A., Leirisalo-Repo, M., Murtomaa, H., \& Meurman, J. H. (2015). Impact of rheumatic diseases on oral health and quality of life. Oral Diseases, 21, 342-348.

Cady, R. J., Glenn, J. R., Smith, K. M., \& Durham, P. L. (2011). Calcitonin gene-related peptide promotes cellular changes in trigeminal neurons and glia implicated in peripheral and central sensitization. Molecular Pain, 7, 94.

Cady, R. J., Denson, J. E., Sullivan, L. Q., \& Durham, P. L. (2014). Dual orexin receptor antagonist 12 inhibits expression of proteins in neurons and glia implicated in peripheral and central sensitization. Neuroscience, 269, 79-92.

Cavalcante, A. L., Siqueira, R. M., Araujo, J. C., Gondim, D. V., Ribeiro, R. A., Quetz, J. S., ... Vale, M. L. (2013). Role of NMDA receptors in the trigeminal pathway, and the modulatory effect of magnesium in a model of rat temporomandibular joint arthritis. European Journal of Oral Science, 121, 573-583.

Chaves, H. V., Ribeiro, R.d. A., de Souza, A. M., Rodrigues e Silva, A. A., Gomes, A. S. Vale, M. L., ... Brito, G. A. (2011). Experimental model of zymosan-induced arthritis in the rat temporomandibular joint: Role of nitric oxide and neutrophils. Journal of Biomedicine and Biotechnology, 2011, 707985.

Clark, A. K., Yip, P. K., Grist, J., Gentry, C., Staniland, A. A., Marchand, F., ... Malcangio, M. (2007). Inhibition of spinal microglial cathepsin S for the reversal of neuropathic pain. Proceedings of the National Academy of Sciences of the United States of America. 104. Proceedings of the National Academy of Sciences of the United States of America (pp. 10655-10660).

da Conceição Rivanor, R. L., Chaves, H. V., do Val, D. R., de Freitas, A. R., Lemos, J. C., Rodrigues, J. A., ... Benevides, N. M. (2014). A lectin from the green seaweed Caulerpa cupressoides reduces mechanical hyper-nociception and inflammation in the rat temporomandibular joint during zymosan-induced arthritis. International Immunopharmacology, 21, 34-43.

Cunha, F. Q., Poole, S., Lorenzetti, B. B., \& Ferreira, S. H. (1992). The pivotal role of tumour necrosis factor alpha in the development of inflammatory hyperalgesia. British Journal of Pharmacology, 107, 660-664.

Denadai-Souza, A., Cenac, N., Casatti, C. A., Câmara, P. R., Yshii, L. M., Costa, S. K., ... Muscará, M. N. (2010). PAR(2) and temporomandibular joint inflammation in the rat. Journal of Dental Research, 89, 1123-1128.

Franceschini, A., Vilotti, S., Ferrari, M. D., van den Maagdenberg, A. M., Nistri, A., \& Fabbretti, E. (2013). TNF $\alpha$ levels and macrophages expression reflect an inflammatory potential of trigeminal ganglia in a mouse model of familial hemiplegic migraine. PLoS One, 8, e52394.

Gondim, D. V., Costa, J. L., Rocha, S. S., Brito, G. A., Ribeiro, R.d. A., \& Vale, M. L. (2012). Antinociceptive and anti-inflammatory effects of the electroacupuncture on experimental arthritis of the rat temporomandibular joint. Canadian Journal of Physiology and Pharmacology, 90, 395-405.

Guan, Z., Hellman, J., \& Schumacher, M. (2016). Contemporary Views on Inflammatory Pain Mechanisms: TRPing Over Innate and Microglial Pathways. F1000 Research. vol. 30, 5 (pii: F1000 Faculty Rev-2425. eCollection 2016).

Guerrero, A. T., Cunha, T. M., Verri, W. A., Jr., Gazzinelli, R. T., Teixeira, M. M., Cunha, F.
Q., \& Ferreira, S. H. (2012). Toll-like receptor 2/MyD88 signaling mediates zymosaninduced joint hypernociception in mice: Participation of TNF- $\alpha$, IL-1 $\beta$ and CXCL1/ KC. European Journal of Pharmacology, 674, 51-57.

Halassa, M. M., Fellin, T., Takano, H., Dong, J. H., \& Haydon, P. G. (2007). Synaptic islands defined by the territory of a single astrocyte. The Journal of Neuroscience, 27, 6473-6477.

Hucho, T., \& Levine, J. D. (2007). Signaling pathways in sensitization: Toward a nociceptor cell biology. Neuron, 53, 365-376.

Liu, Z., Peng, Y., Long, X., Li, J., Jin, K. E., \& Fang, W. (2014). Manual effect between neuropeptides and inflammatory cytokines in neurogenic SMSCs of human temporomandibular joint. Journal of Huazhong University of Science and Technology Medical Science, 34, 602-607.

McMahon, S. B., \& Malcangio, M. (2009). Current challenges in glia-pain biology. Neuron, $64,46-54$.

Mika, J. (2008). Modulation of microglia can attenuate neuropathic pain symptoms and enhance morphine effectiveness. Pharmacological Reports, 60, 297-307.

Milligan, E. D., \& Watkins, L. R. (2009). Pathological and protective roles of glia in chronic pain. Nature Reviews: Neuroscience, 10, 23-36.

Mosser, C. A., Baptista, S., Arnoux, I., \& Audinat, E. (2017). Microglia in CNS development: Shaping the brain for the future. Progress in Neurobiology, S0301-0082 (30126-5).

Nassif, N. J., Al-Salleeh, F., \& Al-Admawi, M. (2003). The prevalence and treatment needs of symptoms and signs of temporomandibular disorders among young adult males. Journal of Oral Rehabilitation, 30, 944-950.

Pena-dos-Santos, D. R., Severino, F. P., Pereira, S. A., Rodrigues, D. B., Cunha, F. Q., Vieira, S. M., ... Clemente-Napimoga, J. T. (2009). Activation of peripheral kappa/ delta opioid receptors mediates 15-deoxy-(Delta12,14)-prostaglandin J2 inducedantinociception in rat temporomandibular joint. Neuroscience, 163, 1211-1219.

Pocock, J. M., \& Kettenmann, H. (2007). Neurotransmitter receptors on microglia. Trends in Neurosciences, 30, 527-535.

Ribeiro, R. A., Vale, M. L., Thomazzi, S. M., Paschoalato, A. B., Poole, S., Ferreira, S. H., \& Cunha, F. Q. (2000). Involvement of resident macrophages and mast cells in the writhing nociceptive response induced by zymosan and acetic acid in mice. European Journal of Pharmacology, 387, 111-118.

Romero-Reyes, M., \& Uyanik, J. M. (2014). Orofacial pain management: Current perspectives. Journal of Pain Research, 21, 99-115.

Ruiz-Miyazawa, K. W., Zarpelon, A. C., Pinho-Ribeiro, F. A., Pavão-de-Souza, G. F., Casagrande, R., \& Verri, W. A., Jr. (2015). Vinpocetine reduces carrageenan-induced inflammatory hyperalgesia in mice by inhibiting oxidative stress, cytokine production and NF-kB activation in the paw and spinal cord. PloS One, 10, e0118942.

Souza, G. R., Talbot, J., Lotufo, C. M., Cunha, F. Q., Cunha, T. M., \& Ferreira, S. H. (2013) Fractalkine mediates inflammatory pain through activation of satellite glial cells. Proceedings of the National Academy of Science of the United States of America, 110, 11193-11198.

Suter, M. R., Wen, Y. R., Decosterd, I., \& Ji, R. R. (2007). Do glial cells control pain? Neuron Glia Biology, 3, 255-268.

Sweitzer, S. M., Schubert, P., \& DeLeo, J. A. (2001). Propentofylline, a glial modulating agent, exhibits antiallodynic properties in a rat model of neuropathic pain. The Journal of Pharmacology and Experimental Therapeutics, 297, 1210-1217.

Takemura, M., Sugiyo, S., Moritani, M., Kobayashi, M., \& Yonehara, N. (2006) Mechanisms of orofacial pain control in the central nervous system. Archives Histology Cytology, 69, 79-100.

do Val, D. R., Bezerra, M. M., Silva, A. A., Pereira, K. M., Rios, L. C., Lemos, J. C., ... Chaves, H. V. (2014). Tephrosia toxicaria Pers. reduces temporomandibular joint inflammatory hypernociception: The involvement of the HO-1 pathway. European Journal of Pain, 18, 1280-1289.

Vega-Avelaira, D., Ballesteros, J. J., \& López-García, J. A. (2013). Inflammation-induced hyperalgesia and spinal microglia reactivity in neonatal rats. European Journal of Pain, 17, 1180-1188.

Villa, G., Ceruti, S., Zanardelli, M., Magni, G., Jasmin, L., Ohara, P. T., \& Abbracchio, M. P. (2010). Temporomandibular joint inflammation activates glial and immune cells in both the trigeminal ganglia and in the spinal trigeminal nucleus. Molecular Pain, $6,89$.

Von Korff, M., Dworkin, S. F., Le Resche, L., \& Kruger, A. (1988). An epidemiologic comparison of pain complaints. Pain, 32, 173-183. 